Modern Physics Letters A

(C) World Scientific Publishing Company

\title{
Theoretical Aspects of Quintom Models
}

\author{
Taotao Qiu \\ Physics Department, Chung-Yuan Christian University, Chung-li, Taiwan 320 \\ qiutt@ihep.ac.cn
}

Received (Day Month Year)

Revised (Day Month Year)

\begin{abstract}
Quintom models, with its Equation of State being able to cross the cosmological constant boundary $w=-1$, turns out to be attractive for phenomenological study. It can not only be applicable for dark energy model for current universe, but also lead to a bounce scenario in the early universe.
\end{abstract}

Keywords: Keyword1; keyword2; keyword3.

PACS Nos.: include PACS Nos.

\section{1. introduction}

For decades of years, the observational data has put strong evidences on existence of Dark Energy. The earliest evidence comes from the observation of Type Ia Supernovae by Supernova Search Team (SST) and Supernova Cosmology Project (SCP) in 1998 which discovered that our universe has been accelerating 1|2. This accelerating requires a kind of negative pressure matter in order to validate the Einstein Gravity. Other observations implies that our universe is nearly flat (with $\Omega_{\text {total }}=0.9996 \pm 0.0199$ where $\Omega_{\text {total }}$ denotes for the total relative energy density of our universe), while the baryon matter and cold dark matter only takes small part of nearly $27 \%$, leaving large occupation for dark energy 3 . Due to this reason, it is of great importance to study the properties of dark energy. However, at the very beginning, people will always ask the question: what is the dark energy?

In the literature, plenty of dark energy candidates has been proposed, see [4] for a review. People often classify these candidates with respect to its Equation of State (EoS) $w=\frac{p}{\rho}$, where $p$ and $\rho$ denotes for the pressure and energy density, respectively. The simplest dark energy candidate is the cosmological constant with energy density being near the vacuum energy $\rho_{\Lambda} \approx\left(10^{-3} \mathrm{eV}\right)^{4}$ without varying with time 5 . This candidate, proposed initially by A.Einstein, however suffers from the severe problem of fine-tuning and coincidence. For this sake, dynamical dark energy models were proposed, among which are Quintessence $(w>-1) \underline{6}$, Phantom

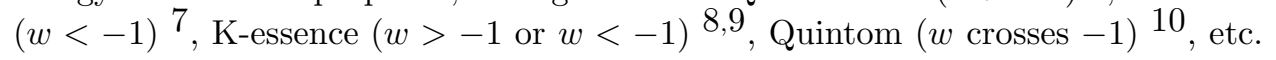


Moreover, it is widely realized that accelerating can also be obtained by modifying the Einstein's Gravity 11. However, the new released data of Supernovae, Wilkinson Microwave Anisotropic Probe observations (WMAP) and Sloan Digital Structure Survey (SDSS) as well as the forthcoming Planck etc., implies that although the cosmological constant with $w=-1$ fits the data well, dynamical candidates still cannot be ruled out. Specifically, the Quintom model whose EoS can cross -1 is mildly favored.

Since the observational data mildly favors Quintom, people may ask: How can we construct such a model theoretically? As is well known, not all of the dark energy models can make it EoS cross -1 which is constrained by the so called "No-Go Theorem" 12|13|14|15|16|17. In this theorem, it is demonstrated that for theory of dark energy, the EoS of dark enegy model will by no means cross the cosmological constant boundary if it is (1) in $4 D$ classical Einstein Gravity, (2) described by single simple component (either perfect fluid or single scalar field with lagrangian as $\left.\mathcal{L}=\mathcal{L}\left(\phi, \partial_{\mu} \phi \partial^{\mu} \phi\right)\right)$, and (3) coupled minimally to Gravity. Indeed, the crossing for such a dark energy model will lead to the divergence of physical quantities, which consequently result in the inconsistency of the system. For sake of this theorem, people proposed various kinds of Quintom models, such as double field Quintom, non-scalar (spinor, vector, etc.) Quintom, Quintom with higher derivative operator, non-minimally coupled Quintom, etc. These models corresponds to violation of different conditions mentioned in the no-go theorem. Some of those models will be reviewed in detail in the next section. Due to the behavior of EoS of Quintom models, it can as well lead to various evolution of the universe which cannot be realized by non-Quintom dark energy models.

Furthermore, if we apply the property of Quintom to the early universe, some interesting features will also be expected, for example, a bounce scenario will be obtained 18. A Quintom model will give rise to a bounce scenario in $4 D$ Einstein Gravity, i.e. there is no need to introduce extra dimensions. This can be seen by investigating the conditions for a bounce to happen. In a contracting phase the scale factor is decreasing, i.e., $a(t)<0$, while in an expanding phase the scale factor is increasing, $a \dot{a(t)}>0$. Therefore we expect that at the transition point $a \dot{(t)}=0$ while $a \ddot{(t})>0$. Equivalently, we expect the Hubble parameter $H$ cross the zero point from $H<0$ to $H>0$ at the bouncing point, which requires the EoS of the universe less than -1 according to the Einstein Equation. After the bounce, in order for the universe to enter into the realistic one with matter dominating, radiation dominating, etc., it is required that the EoS of the universe being larger than -1 . That is, the EoS will cross -1 during the whole bounce process, and a Quintom behavior is needed.

Based on the scenario of Quintom bounce, an important problem is that how can it give rise to the observed amount of perturbation to form the large scale structures? To answer this question, we constructed the perturbation theory of Quintom bounce 1920. In our model, Quintom field can act as inflaton after bounce and drive enough period of inflation. In this scenario, the inflation stage can dilute everything away 
and set the universe to be near the state of the universe after the standard inflation, so the bounce process may hardly effect the following evolution of the universe and fits well with all the current data. Nevertheless, we do expect that bounce can leave some signature that can be seen for future observations. Furthurmore, using Quintom field we can also build another bounce model known as "Lee-Wick type"

or "matter bounce", which requires no inflationary stage at all 21. In this scenario, the perturbation is also calculated and a scale invariant power spectrum is produced during the contraction of the universe.

\section{2. the Quintom model}

Quintom model was initially proposed in [10, where the authors combined the Quintessence and Phantom components together. The typical action of these kind of model is:

$$
S_{\text {Quintom }}=\int d^{4} x \sqrt{-g}\left\{\frac{1}{2} \nabla_{\mu} \phi_{1} \nabla^{\mu} \phi_{1}-\frac{1}{2} \nabla_{\mu} \phi_{2} \nabla^{\mu} \phi_{2}-V\left(\phi_{1}, \phi_{2}\right)\right\},
$$

where $\phi_{1}$ and $\phi_{2}$ are Quintessence and Phantom components, respectively. Due to the combined effects of the two, it is an intuition to see that the total EoS of the whole system will evolve across the cosmological constant boundary. Generally, the effective potential can be of arbitrary form, while the two components can be either coupled or decoupled. In the original paper 10 , the authors considered a simple form of decoupled case: $V\left(\phi_{1}, \phi_{2}\right)=V_{0}\left(e^{-\frac{\lambda \phi_{1}}{m_{p l}}}+e^{-\frac{\lambda \phi_{2}}{m_{p l}}}\right)$ where $m_{p l}$ denotes for the Planck mass while $\lambda$ is dimensionless constant. The evolution of the EoS was plotted in Fig. 1.

After the first Quintom paper came up, lots of people investigated its property due to its importance. In [22, the attractor solution has been studied and in 223, people extended to the more general case of which a coupling term has been introduced. See also [24] for more variety of double-scalar Quintom models. In [15], the perturbation of Quintom model has been calculated and a self-consistent perturbation theory were constructed.

Double field is the simplest and most natural scenario of Quintom model. However, it suffers from many problems such as big-rip and quantum instability. So people have to think about alternative ways to realize the EoS crossing. Another Quintom model is the addition of a higher derivative operator to the single scalar field 25. The most general lagrangian is as follows:

$$
\mathcal{L}=\mathcal{L}\left(\phi, \nabla_{\mu} \phi \nabla^{\mu} \phi, \square \phi, \nabla_{\mu} \nabla_{\nu} \phi \nabla^{\mu} \nabla^{\nu} \phi, \ldots\right),
$$

where $\square=\nabla_{\mu} \nabla^{\mu}$ is the d'Alembertian operator and the ellipse denotes for other higher dimensional operators. The higher order operators can be derived from fundamental theories such as string theory or quantum gravity $26|27| 28$, and with the addition of high order terms to the Einstein Gravity, the theory is shown to be renormalizable 29. Because of the extra degrees of freedom provided by the higher order term, it can simulate double-field model in some specific cases. However, it 
has more interesting features of its own. In Higher derivative theory, the dispersion relation is modified, and it may provide possible solutions to the problem of quantum instability 26/30. Furthermore, because it is more complex and involved in the high derivative term, it may give rise to some new behaviors of evolution of the universe. 31.

We consider the phenomenological form of higher derivative action as follows

$$
\mathcal{S}_{H D}=\int d^{4} x \sqrt{-g}\left\{A(\phi) \nabla_{\mu} \phi \nabla^{\mu} \phi-\frac{C(\phi)(\square \phi)^{2}}{m_{p l}^{2}}-V(\phi)\right\},
$$

where $A(\phi)$ and $C(\phi)$ are some functions of the field $\phi$. For various choice of the form of these functions, the evolution of the field could be very different. For sake of simplicity, we choose $A(\phi)=-\frac{1}{2}$ and $V(\phi)=0$ as an example. By redefining the field variable $\chi=\frac{C(\phi)}{m_{p l}^{2}} \square \phi$ and $\psi=-(\phi+\chi)$, we obtain a simple form of action:

$$
\mathcal{S}_{\text {eff }}=\int d^{4} x \sqrt{-g}\left\{-\frac{1}{2} \nabla_{\mu} \psi \nabla^{\mu} \psi+\frac{1}{2} \nabla_{\mu} \chi \nabla^{\mu} \chi-\frac{m_{p l}^{2} \chi^{2}}{C(\psi+\chi)}\right\},
$$

and the last term could be viewed as an effective mass term of $\chi$ which varies in terms of $\psi$ and $\chi$. Here we can see, $\chi$ acts as a normal field while $\psi$ being the "ghost" field with a wrong sign. We can choose the form of $C(\phi+\chi)$ to control the evolution of the two field, so that the EoS of the whole system can not only cross -1 , but also present novel behaviors. In our numerical analysis, we choose $C(\phi+\chi)$ to be small at the beginning and large at the end of the evolution, both of the two region being nearly a constant, while in the mediate region it has a significantly running. Therefore, at the beginning of evolution the "ghost" field $\psi$ evolves as a massless field with its own effective EoS nearly unity. In the mediate region, however, the effect of $\psi$ in potential term is involked and it behaves like a real Phantom and draw the total EoS below -1. In the future, it behaves like massless field again. While in this region the normal field $\chi$ gets a large value of effective mass, the whole system will evolve as a non-relativistic matter with the EoS oscillating around zero. See numerical results in Fig. 2 ,

For actions of field theory linear with the kinetic term, higher derivative operator could only exist as higher order term with an energy cut-off. However, it can also exist with the same order as the kinetic term in a non-linear field theory. An explicit example is the so-called "String Inspired Quintom", where such a term resides in the square root in the Dirac-Born-Infeld (DBI) action 32. The effective action is given as:

$$
\mathcal{S}_{D B I}=\int d^{4} x \sqrt{-g}\left\{-V(\phi) \sqrt{1-\alpha^{\prime} \nabla_{\mu} \phi \nabla^{\mu} \phi+\beta^{\prime} \phi \square \phi}\right\},
$$

where $\alpha^{\prime}$ and $\beta^{\prime}$ are coefficients of dimension 4 . One can see that without the high derivative term, the action will be reduced to the normal DBI action describing a tachyon state in string theory 33 . However, one cannot remove this term by adding 
a total integral to the action as in the linear theory. Furthermore, one can also include infinite numbers of higher order derivative terms as in the context of p-adic string theory 34 .

Due to the effect of the higher derivative operator, one can see from the numerical results in Fig. 3 that the EoS can also cross -1 naturally and various behaviors are presented according to the form of the potential. As a non-linear theory, it is also important to check the stability of classical perturbation. We calculated the second order action of this model and numerically obtained the variation of $c_{s}^{2}$ with respect to time during evolution. From the result one could see that for our cases $c_{s}^{2}$ varies between the range of $(0,1)$, denoting neither instability nor unphysical propagation of the fluctuation.

Of course there are other models that can make EoS crossing -1, among which are vector fields 35 , spinor fields 36 , non-minimal coupling fields 37 , as well as the theories of modified gravity or high dimensional theories 38/39, which will not be discussed here because of the page limit.

As a side remark, it is noticeable that due to the dynamic behavior of Quintom models, it can lead to many interesting fates of the universe in the future, which is expected to be determined by forthcoming observations. The asymptotic behavior of Quintom can mimic that of Quintessence, Phantom, as well as $\Lambda$ CDM model. Furthermore, it can bring novel features that cannot be realized by any of them, for instance, the oscillating behavior around the cosmological constant boundary. Within this phenomenon, we could construct a cyclic or recurrent universe 40|41. Other features include that the Quintom models has a cosmic self-duality where one kind of Quintom model in expanding universe is dual to the other in contracting universe depending on the initial conditions 42 . In this sense, Quintom model is of very much interest in phenomenology. Meanwhile, there are also many subtle issues about Quintom that remains unclear, such as its connection to the fundemental theories or particle physics, and its nature in quantum levels, etc., which are worthwhile of investigation in the future.

\section{Quintom bounce story: background and perturbation}

For decades of years, the theory of inflation has attracted many attentions for its success in solving most problems (flatness problem, horizon problem, etc) that arised in Standard Big-Bang Cosmology 43 . However, inflation is far from a complete theory since it suffers from other problems such as singularity problem 44 and transplanckian problem 45. Due to this reason, people proposed several alternative solutions of the early universe, among which are Pre-Big-Bang scenario 46, Ekpyrotic scenario 47, string gas scenario 48 , non-local string field theory scenario 49 and so on. When restricted to $4 \mathrm{D}$ effective theory, a simple way to get rid of the singularity is to have a bounce process at the early stage. It can be realized by a model of ghost condensate $56 \mid 57$ or the modification of Einstein Gravity $50|51 / 52| 53154 \mid 55$.

Quintom model, as mentioned in the introduction, can also provide a bounce 
solution of the early universe, avoiding the singularity naturally. As an example, In Fig. 4 we draw a phenomenological parametrization of Quintom model. From the picture we can see that as the EoS evolves, the scale factor of the universe can transfer from damping to growing, i.e., a bounce happens.

In order to compare the predictions of Quintom bounce scenario to the observations, it is necessary to investigate the perturbations of the scenario. Indeed, to make the scenario realistic, one need the perturbations after the bounce as seeds of forming our galaxies and large scale structures. In singular bounce scenarios such as Pre Big Bang and Ekpyrosis, the fluctuation cannot evolve through the bounce point consistently. It will diverge at the pivot and thus invalidate the linear perturbation theory. While in our scenario, as we will see, the proper matching condition can be used and the fluctuations can transfer from contraction to expansion naturally. Furthermore, according to different mode, the fluctuation can possess features both found in singular and non-singular bouncing models. The power spectrum of our scenario has also been calculated, and due to different models, we can get either running or scale invariant power spectrum $19 \mid 20$.

In what follows, we will focus on bounce scenario caused by non-interacting double field Quintom. The general form of such a model is:

$$
\mathcal{L}_{Q B}=\frac{1}{2} \nabla_{\mu} \phi \nabla^{\mu} \phi-\frac{1}{2} \nabla_{\mu} \psi \nabla^{\mu} \psi-V(\phi)-W(\psi),
$$

where $V(\phi)$ and $W(\psi)$ are potentials for normal and ghost fields. We will see that due to different forms of potentials, different results will come about.

Case I. $V(\phi)=\frac{1}{2} m^{2} \phi^{2}, W(\psi)=0$.

In this case 19, the ghost field only remains its kinetic term, thus its energy density evolves proportional to $a^{-6}$ and become important only near the bouncing point. At regions far away from the bounce, the universe is dominated by the normal field. We begin the contracting phase with $\phi$ oscillating around the minimum of the potential. Because of the contraction of the universe, the amplitude of the oscillation grows as $a^{3 / 2}$ and the field behaves as non-realistic matter. This period is called "Heating phase". After the last oscillation, the field climb up along the potential and caused a period of "deflation" of which the EoS of the universe approximately near -1 . Meanwhile, the energy density of the field $\psi$ is growing all the time. When it catches up with that of the field $\phi$, the total energy density vanishes. As we learn from Einstein Equation that $H=0$ with a positive time derivative $\dot{H}$. Thus the bounce happens. After the bounce the $\psi$ field damps quickly while the $\phi$ field rolls down slowly along its potential, very much like the chaotic inflation model. At last, $\phi$ oscillates around the minimum of the potential again, with a damping amplitude.

The left hand side of Fig. 5 is the evolution of EoS during the bounce process and the right hand side is the sketch plot of the space-time in bounce scenario while the horizonal and vertical axis denotes for physical distance and time respectively. The black solid line represents the Hubble horizon and green and blue line are different fluctuation modes. 
From perturbed Einstein Equation, we get the equation for the Newtonian gravitational potential $\Phi$ as 58 .

$$
\Phi^{\prime \prime}+2\left(\mathcal{H}-\frac{\phi^{\prime \prime}}{\phi^{\prime}}\right) \Phi^{\prime}+2\left(\mathcal{H}^{\prime}-\mathcal{H} \frac{\phi^{\prime \prime}}{\phi^{\prime}}\right) \Phi-\nabla^{2} \Phi=8 \pi G\left(2 \mathcal{H}+\frac{\phi^{\prime \prime}}{\phi^{\prime}}\right) \psi \delta \psi^{\prime},
$$

where $\mathcal{H}$ is the comoving Hubble constant, and prime denotes derivative with respect to comoving time $\eta$. For the initial condition, we set it as Bunch-Davies vacuum as usual in the far past, when $u \equiv \frac{a \Phi}{\phi^{\prime}} \sim \frac{1}{\sqrt{2 k^{3}}}$ and $\Phi \sim \eta^{-3} \frac{1}{\sqrt{2 k^{3}}}$. Here we use the fact that at the very beginning when $w \approx 0$ we have $\frac{\phi^{\prime}}{a} \sim \eta^{-3}$. At regions far from bouncing point, the right hand side of Eq. (7) can be neglected and the equation becomes homogeneous. It has two branches of solution at super-Hubble region, one is growing while the other is constant. At the regions near the bounce, we get both of the branches oscillating with the amplitude depending on the energy scale of the bouncing. After the bounce, we again get two branches where one is constant while the other is damping. The solutions of each stage is matched consistently via Deruelle-Mukhanov matching conditions 5960 .

Unfortunately, this kind of model cannot give rise to a scale invariant power spectrum as obtained by observation. The reason is easy to see: In the contracting phase, the perturbation will get out of the horizon and the amplitude of the perturbation will be raised by the growing mode. Such a behavior corresponds to the modification of the initial condition of perturbations at the following inflationary stage. The power spectrum will thus have a blue tilt. For the above reason, some alternative models need to be considered.

Case II: $V(\phi)=\frac{\lambda \phi^{4}}{4}\left(\ln \frac{|\phi|}{v}-\frac{1}{4}\right)+\frac{\lambda v^{4}}{16}, W(\psi)=0$.

In this case 20 , the potential of normal field $\phi$ is of Coleman-Weinberg type, with two vacua set on both sides of the middle $\frac{61}{\text {, }}$, see Fig. 6. We set our initial state with $\phi$ residing in one of the vacua while oscillating. When the amplitude grows large enough the field climbs to the plateau of the potential, while the growing energy density of the second field $\psi$ catches up, and the bounce happens, followed by a period of inflation. As one could see from the numerical analysis of background evolution that, there is no "deflation" period in the contracting phase and the symmetry with respect to the bouncing point has been broken, see Fig. 7 . Thanks to this symmetry violation, we could see from the sketch plot that for large $k$ modes, the fluctuation will stay inside the horizon all the contracting time, leading to a scale invariant power spectrum. Meanwhile, for small $k$ modes which exits horizon during contraction, the spectrum will have a blue tilt.

Case III: $V(\phi)=\frac{1}{2} m^{2} \phi^{2}, W(\psi)=-\frac{1}{2} M^{2} \psi^{2}$.

This is another interesting case where both of the field get a mass term, while that of the ghost field has a wrong sign 21. This model can be obtained from the scalar sector of Lee-Wick Standard Model with $M \gg m$ 62. In this case, both of the fields start off oscillating around their extremes of the potential, and the initial stage is dominated by the normal field. The oscillation amplitude of both fields scale as $a(t)^{-3 / 2}$ and the universe presents non-relativistic-matter-like. However, $\psi$ oscillates 
much rapidly due to the heavy mass, and eventually the energy density of $\psi$ catches up with $\phi$ and bounce happens. After the bounce, both fields continues oscillating with a damping amplitude. During the whole process except the bouncing point, the EoS of the universe has an average value of $w=0$ and there is neither deflation nor inflation era at all. See Fig. 8 ,

In this model, a scale invariant power spectrum can be obtained during contracting phase for fluctuations on scales larger than Hubble radius. This could be seen easily if we work in terms of the comoving curvature perturbation $\zeta$, or equivalently, the Mukhanov-Sasaki variable $v=z \zeta \frac{6364}{6 h e r e ~} z \sim a$ for time-independent EoS. The well-known equation of motion for $v$ is:

$$
v^{\prime \prime}+\left(k^{2}-\frac{z^{\prime \prime}}{z}\right) v=0 .
$$

For super-Hubble fluctuation modes, one can neglect the $k^{2}$ term and make use of the parametrization $a(\eta) \sim \eta^{2}$ for $w=0$ to get $v \sim \eta^{-1}$, and hence obtain the $k$-dependence of power spectrum:

$$
P_{\zeta}(k, \eta) \sim k^{3}|v(\eta)|^{2} a(\eta)^{-2} \sim k^{3}\left|v\left(\eta_{H}(k)\right)\right|^{2}\left(\frac{\eta_{H}(k)}{\eta}\right)^{2} \sim k^{3-1-2} \sim k^{0} .
$$

In deriving the above formula we've used the fact that the comoving time when fluctuations cross the Hubble horizon $\eta_{H}(k) \sim k^{-1}$.

\section{4. summary}

This talk is mainly focused on the properties of Quintom dark energy model and its application to the early universe. Quintom model, which needs multi degrees of freedom, has an EoS crossing -1 and can bring various features of the universe in the future. When applying to the early universe, it can give rise to a bouncing scenario. The perturbation theory of Quintom bounce is self consistent and a scale invariant power spectrum can be obtained.

\section{Acknowledgments}

It is a pleasure to thank the organizers of "The International Workshop on Dark Matter, Dark Energy and Matter-antimatter Asymmetry" for the invitation to speak and for their wonderful hospitality in National Tsinghua University, Taiwan. I also wish to thank Prof. R. Brandenberger, Yifu Cai, Prof. Yunsong Piao, Prof. Hong Li, Prof. Mingzhe Li, Jie Liu, Dr. Junqing Xia, Prof. Xinmin Zhang, Dr. Xiaofei Zhang and Dr. Gongbo Zhao for useful help. and all the authors of the figures for allowing me to use their figures as a citation. My research is supported in parts by the National Science Council of R.O.C. under Grant No. NSC96-2112-M-033004-MY3 and No. NSC97-2811-033-003 and by the National Center for Theoretical Science. 


\section{References}

1. A. G. Riess et al. [Supernova Search Team Collaboration], Astron. J. 116, 1009 (1998) arXiv:astro-ph/9805201.

2. S. Perlmutter et al. [Supernova Cosmology Project Collaboration], Astrophys. J. 517, 565 (1999) arXiv:astro-ph/9812133.

3. E. Komatsu et al. [WMAP Collaboration], Astrophys. J. Suppl. 180, 330 (2009) arXiv:0803.0547 [astro-ph]].

4. E. J. Copeland, M. Sami and S. Tsujikawa, Int. J. Mod. Phys. D 15, 1753 (2006) arXiv:hep-th/0603057.

5. S. Weinberg, Rev. Mod. Phys. 61, 1 (1989).

6. B. Ratra and P. J. E. Peebles, Phys. Rev. D 37, 3406 (1988).

7. R. R. Caldwell, Phys. Lett. B 545, 23 (2002) arXiv:astro-ph/9908168.

8. T. Chiba, T. Okabe and M. Yamaguchi, Phys. Rev. D 62, 023511 (2000) arXiv:astro-ph/9912463.

9. C. Armendariz-Picon, V. F. Mukhanov and P. J. Steinhardt, Phys. Rev. Lett. 85, 4438 (2000) arXiv:astro-ph/0004134.

10. B. Feng, X. L. Wang and X. M. Zhang, Phys. Lett. B 607, 35 (2005) arXiv:astro-ph/0404224.

11. S. Capozziello, S. Carloni and A. Troisi, Recent Res. Dev. Astron. Astrophys. 1, 625 (2003) arXiv:astro-ph/0303041.

12. A. Vikman, Phys. Rev. D 71, 023515 (2005) arXiv:astro-ph/0407107.

13. W. Hu, Phys. Rev. D 71, 047301 (2005) arXiv:astro-ph/0410680].

14. R. R. Caldwell and M. Doran, Phys. Rev. D 72, $043527 \quad$ (2005) arXiv:astro-ph/0501104.

15. G. B. Zhao, J. Q. Xia, M. Li, B. Feng and X. Zhang, Phys. Rev. D 72, 123515 (2005) arXiv:astro-ph/0507482.

16. M. Kunz and D. Sapone, Phys. Rev. D 74, 123503 (2006) arXiv:astro-ph/0609040.

17. J. Q. Xia, Y. F. Cai, T. T. Qiu, G. B. Zhao and X. Zhang, Int. J. Mod. Phys. D 17, 1229 (2008) arXiv:astro-ph/0703202.

18. Y. F. Cai, T. Qiu, Y. S. Piao, M. Li and X. Zhang, JHEP 0710, 071 (2007) arXiv:0704.1090 [gr-qc]].

19. Y. F. Cai, T. Qiu, R. Brandenberger, Y. S. Piao and X. Zhang, JCAP 0803, 013 (2008) arXiv:0711.2187 [hep-th]].

20. Y. F. Cai, T. t. Qiu, J. Q. Xia and X. Zhang, Phys. Rev. D 79, 021303 (2009) arXiv:0808.0819 [astro-ph]].

21. Y. F. Cai, T. t. Qiu, R. Brandenberger and X. m. Zhang, Phys. Rev. D 80, 023511 (2009) arXiv:0810.4677 [hep-th]].

22. Z. K. Guo, Y. S. Piao, X. M. Zhang and Y. Z. Zhang, Phys. Lett. B 608, 177 (2005) arXiv:astro-ph/0410654.

23. X. F. Zhang, H. Li, Y. S. Piao and X. M. Zhang, Mod. Phys. Lett. A 21, 231 (2006) arXiv:astro-ph/0501652.

24. Y. F. Cai, E. N. Saridakis, M. R. Setare and J. Q. Xia, arXiv:0909.2776 [hep-th].

25. M. z. Li, B. Feng and X. m. Zhang, JCAP 0512, 002 (2005) arXiv:hep-ph/0503268.

26. J. Z. Simon, Phys. Rev. D 41, 3720 (1990).

27. D. A. Eliezer and R. P. Woodard, Nucl. Phys. B 325, 389 (1989).

28. T. Erler and D. J. Gross, arXiv:hep-th/0406199.

29. K. S. Stelle, Phys. Rev. D 16, 953 (1977).

30. S. W. Hawking and T. Hertog, Phys. Rev. D 65, 103515 (2002) arXiv:hep-th/0107088.

31. X. F. Zhang and T. Qiu, Phys. Lett. B 642, 187 (2006) arXiv:astro-ph/0603824. 
32. Y. f. Cai, M. z. Li, J. X. Lu, Y. S. Piao, T. t. Qiu and X. m. Zhang, Phys. Lett. B 651, 1 (2007) arXiv:hep-th/0701016.

33. A. Sen, JHEP 0204, 048 (2002) arXiv:hep-th/0203211].

34. N. Barnaby, T. Biswas and J. M. Cline, JHEP 0704, 056 (2007) arXiv:hep-th/0612230.

35. C. Armendariz-Picon, JCAP 0407, 007 (2004) arXiv:astro-ph/0405267.

36. Y. F. Cai and J. Wang, Class. Quant. Grav. 25, 165014 (2008) arXiv:0806.3890 [hepth]].

37. L. Perivolaropoulos, JCAP 0510, 001 (2005) arXiv:astro-ph/0504582.

38. I. Y. Aref'eva, A. S. Koshelev and S. Y. Vernov, Phys. Rev. D 72, 064017 (2005) arXiv:astro-ph/0507067.

39. H. S. Zhang and Z. H. Zhu, Phys. Rev. D 75, 023510 (2007) arXiv:astro-ph/0611834.

40. B. Feng, M. Li, Y. S. Piao and X. Zhang, Phys. Lett. B 634, 101 (2006) arXiv:astro-ph/0407432.

41. H. H. Xiong, Y. F. Cai, T. Qiu, Y. S. Piao and X. Zhang, Phys. Lett. B 666, 212 (2008) arXiv:0805.0413 [astro-ph]].

42. Y. f. Cai, H. Li, Y. S. Piao and X. m. Zhang, Phys. Lett. B 646, 141 (2007) arXiv:gr-qc/0609039.

43. A. H. Guth, Phys. Rev. D 23, 347 (1981).

44. A. Borde and A. Vilenkin, Phys. Rev. Lett. 72, 3305 (1994) arXiv:gr-qc/9312022.

45. J. Martin and R. H. Brandenberger, Phys. Rev. D 63, 123501 (2001) arXiv:hep-th/0005209.

46. M. Gasperini and G. Veneziano, Astropart. Phys. 1, 317 (1993) arXiv:hep-th/9211021.

47. J. Khoury, B. A. Ovrut, P. J. Steinhardt and N. Turok, Phys. Rev. D 64, 123522 (2001) arXiv:hep-th/0103239.

48. R. H. Brandenberger and C. Vafa, Nucl. Phys. B 316, 391 (1989).

49. I. Y. Aref'eva, L. V. Joukovskaya and S. Y. Vernov, JHEP 0707, 087 (2007) arXiv:hep-th/0701184.

50. R. Brustein and R. Madden, Phys. Rev. D 57, 712 (1998) arXiv:hep-th/9708046.

51. C. Cartier, E. J. Copeland and R. Madden, JHEP 0001, 035 (2000) arXiv:hep-th/9910169.

52. S. Tsujikawa, R. Brandenberger and F. Finelli, Phys. Rev. D 66, 083513 (2002) arXiv:hep-th/0207228].

53. T. Biswas, A. Mazumdar and W. Siegel, JCAP 0603, 009 (2006) arXiv:hep-th/0508194.

54. T. Biswas, R. Brandenberger, A. Mazumdar and W. Siegel, JCAP 0712, 011 (2007) arXiv:hep-th/0610274.

55. Y. F. Cai and E. N. Saridakis, JCAP 0910, 020 (2009) arXiv:0906.1789 [hep-th]].

56. P. Creminelli and L. Senatore, JCAP 0711, 010 (2007) arXiv:hep-th/0702165.

57. E. I. Buchbinder, J. Khoury and B. A. Ovrut, Phys. Rev. D 76, 123503 (2007) arXiv:hep-th/0702154.

58. V. F. Mukhanov, H. A. Feldman and R. H. Brandenberger, Phys. Rept. 215, 203 (1992).

59. J. c. Hwang and E. T. Vishniac, Astrophys. J. 382, 363 (1991).

60. N. Deruelle and V. F. Mukhanov, Phys. Rev. D 52, 5549 (1995) arXiv:gr-qc/9503050.

61. S. R. Coleman and E. J. Weinberg, Phys. Rev. D 7, 1888 (1973).

62. B. Grinstein, D. O'Connell and M. B. Wise, Phys. Rev. D 77, 025012 (2008) arXiv:0704.1845 [hep-ph]].

63. M. Sasaki, Prog. Theor. Phys. 76, 1036 (1986). 
64. V. F. Mukhanov, Sov. Phys. JETP 67 (1988) 1297 [Zh. Eksp. Teor. Fiz. 94N7 (1988) 1].

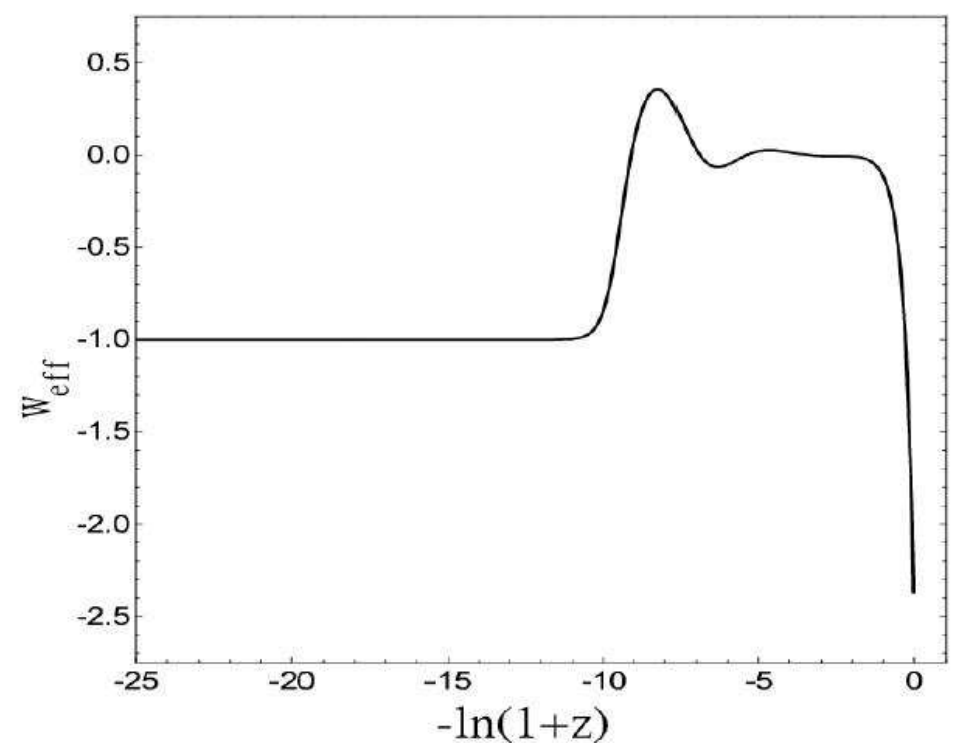

Fig. 1. The evolution of the effective EoS of double-field Quintom model proposed in [10]. The parameters are chosen as: $V_{0}=8.38 \times 10^{-126} m_{p l}^{4}, \lambda=20$. The initial conditions were chosen as: $\phi_{1 i}=-1.7 m_{p l}, \phi_{2 i}=-0.2292 m_{p l}$.
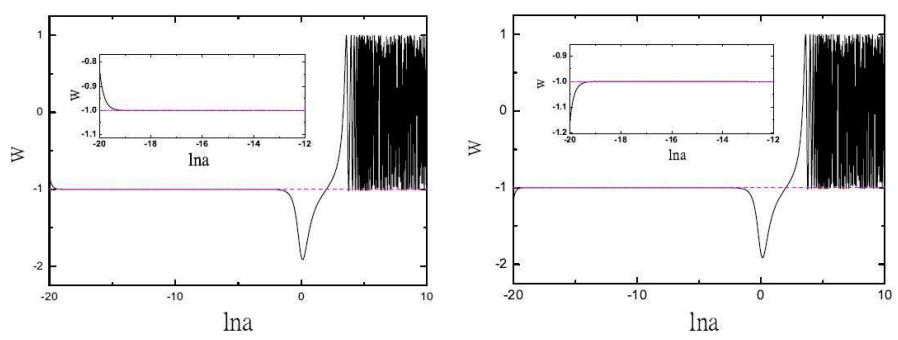

Fig. 2. The evolution of EoS of the model proposed in [31]. For the left hand side case the initial values are $\psi_{i}=-0.26 m_{p l}, \dot{\psi}_{i}=3.52 \times 10^{-62} m_{p l}^{2}, \chi_{i}=0.25 m_{p l}, \dot{\chi}_{i}=-3.62 \times 10^{-62} m_{p l}^{2}$, while for the right hand side case they are $\psi_{i}=-0.26 m_{p l}, \dot{\psi}_{i}=-2.84 \times 10^{-62} m_{p l}^{2}, \chi_{i}=0.25 m_{p l}$, $\dot{\chi}_{i}=2.74 \times 10^{-62} m_{p l}^{2}$. 

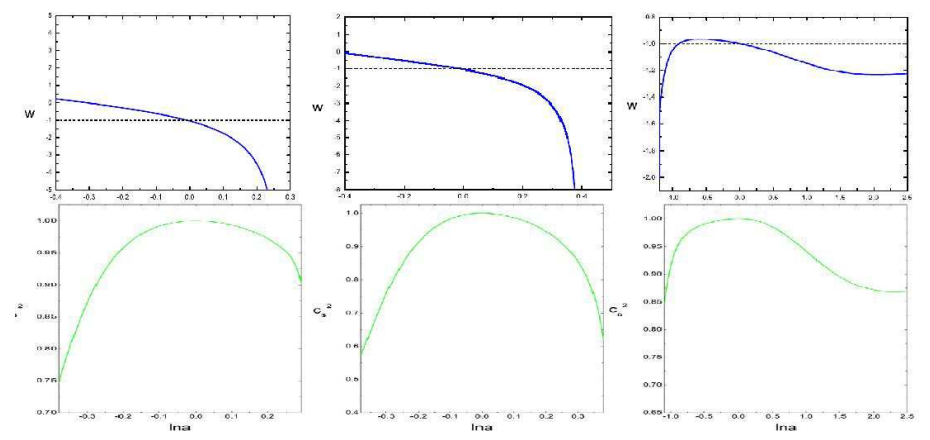

Fig. 3. The first row: The evolution of the EoS of the "String Inspired Quintom model", of which the first picture for potential $V(\phi)=V_{0} e^{-\lambda \phi^{2}}$ while the last two for potential $V(\phi)=\frac{V_{0}}{e^{\lambda \phi}+e^{-\lambda \phi}}$ and different parameter choices. The second row: The variation of the corresponding $c_{s}^{2}$ of each case obove.
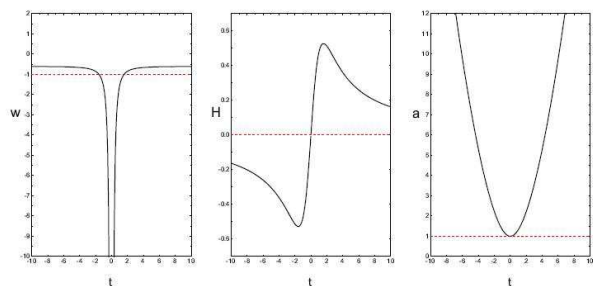

Fig. 4. The evolution of the $\operatorname{EoS} w$, hubble parameter $H$ and the scale factor $a$ as a function of the cosmic time $t$. The EoS is parameterized as $w=-r-\frac{s}{t^{2}}$ where $r=0.6$ and $s=1$.
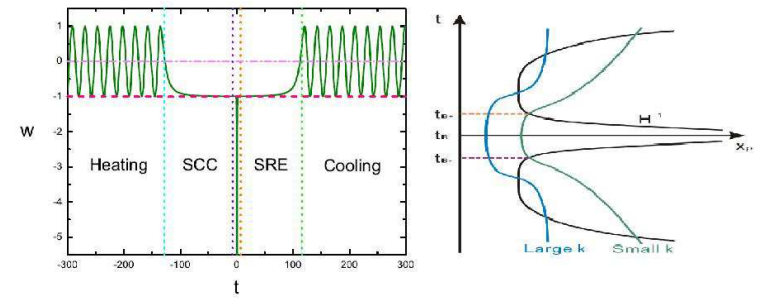

Fig. 5. The left hand side: The evolution of the EoS in Quintom bounce model of Case I. The initial values are chosen as $\phi_{i}=-5.6 \times 10^{-3} m_{p l}, \dot{\phi}_{i}=2.56 \times 10^{-10} m_{p l}^{2}, \dot{\psi}_{i}=4.62 \times 10^{-85} m_{p l}^{2}$ with $m=1.414 \times 10^{-7} m_{p l}$. The right hand side: A sketch of the evolution of perturbations with different comoving wave numbers $k$ in this case. 


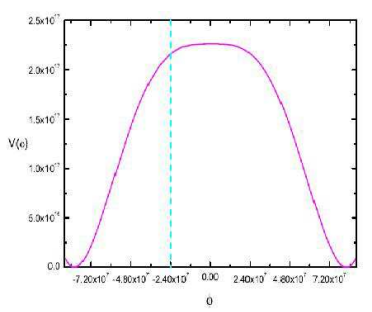

Fig. 6. The sketch plot of the potential as function of $\phi$ in Quintom bounce model of Case II.

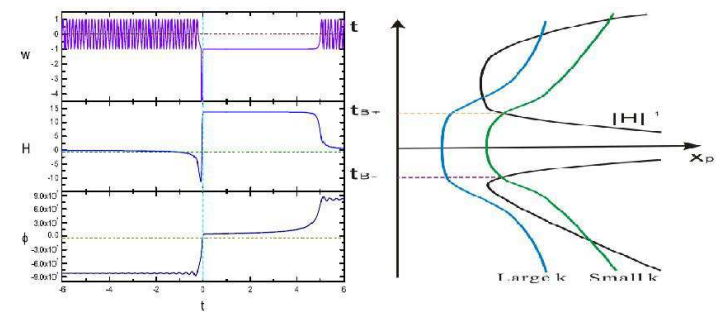

Fig. 7. The left hand side: The evolution of the EoS in Quintom bounce model of Case II. The initial values are chosen as $\phi_{i}=-0.82 m_{p l}, \dot{\phi}_{i}=3.0 \times 10^{-10} m_{p l}^{2}, \dot{\psi}_{i}=5.0 \times 10^{-13} m_{p l}^{2}$ with $\lambda=8.0 \times 10^{-14}$ and $v=0.82 m_{p l}$. The right hand side: A sketch of the evolution of perturbations with different comoving wave numbers $k$ in this case.

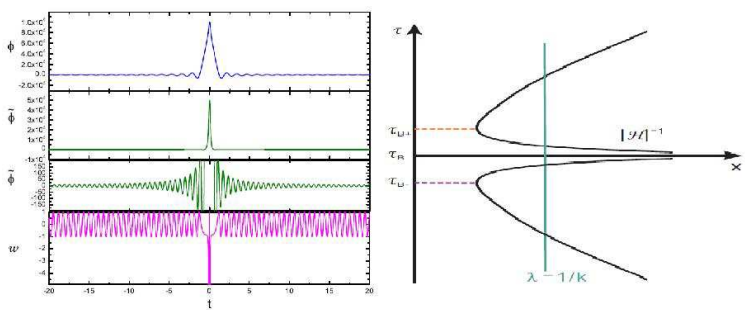

Fig. 8. The left hand side: The evolution of the EoS in Quintom bounce model of Case III. The initial values are chosen as $\phi_{i}=1.74 \times 10^{-3} m_{p l}, \dot{\phi}_{i}=1.44 \times 10^{-8} m_{p l}^{2}, \psi_{i}=8.98 \times 10^{-6} m_{p l}$, $\dot{\psi}_{i}=-14.08 \times 10^{-12} m_{p l}^{2}$ with $m=5.0 \times 10^{-6} m_{p l}, M=1.0 \times 10^{-5} m_{p l}$. The right hand side: A sketch of the evolution of perturbations with different comoving wave numbers $k$ in this case. 